\title{
Differences of Fractional Order
}

\author{
By J. B. Diaz and T. J. Osler
}

\begin{abstract}
Derivatives of fractional order, $D^{\alpha}$, have been considered extensively in the literature. However, little attention seems to have been given to finite differences of fractional order, $\Delta^{\alpha} f$. In this paper, a definition of differences of arbitrary order is presented, and $\Delta^{\alpha} f$ is computed for several specific functions $f$ (Table 2.1). We find that the operator $\Delta^{\alpha}$ is closely related to the contour integral which defines Meijer's $G$-function. A Leibniz rule for the fractional difference of the product of two functions is discovered and used to generate series expansions involving the special functions.
\end{abstract}

1. Introduction. It is well known that there is a remarkable similarity between formulas from the differential calculus, involving the operator $D=d / d z$, and formulas from the calculus of finite differences, involving the operator $\Delta$, which we define by $\Delta f(z)=f(z+1)-f(z)$.

In particular, we recall the following similar pairs of formulas [3]:

$$
\begin{gathered}
D z^{p}=p z^{n-1}, \quad \Delta z^{(p)}=p z^{(p-1)} ; \\
D^{n} z^{p}=\frac{p ! z^{p-n}}{(p-n) !}, \quad \Delta^{n} z^{(p)}=\frac{p ! z^{(p-n)}}{(p-n) !} \\
D^{n} a^{z}=(\log a)^{n} a^{z}, \quad \Delta^{n} a^{z}=(a-1)^{n} a^{2} ; \\
D^{n} D^{m} f(z)=D^{n+m} f(z), \quad \Delta^{n} \Delta^{m} f(z)=\Delta^{n+m} f(z) ; \\
f(z)=\sum_{n=0}^{\infty} \frac{D^{n} f(a)}{n !}(z-a)^{n}, \quad f(z)=\sum_{n=0}^{\infty} \frac{\Delta^{n} f(a)}{n !}(z-a)^{(n)} ; \\
\int_{m}^{n} D f(z) d z=f(n)-f(m), \quad \sum_{m}^{n} \Delta f(z)=f(n+1)-f(m) ; \\
D^{n} f(z) g(z)=\sum_{k=0}^{\infty}\left(\begin{array}{l}
n \\
k
\end{array}\right) D^{n-k} f(z) D^{k} g(z), \\
\Delta^{n} f(z) g(z)=\sum_{k=0}^{\infty}\left(\begin{array}{l}
n \\
k
\end{array}\right) \Delta^{n-k} f(z) \Delta^{k} g(z+n-k) .
\end{gathered}
$$

In these formulas, the notation $z^{(p)}=z(z-1)(z-2) \cdots(z-p+1)$ is used, with $p=0,1,2, \cdots$. We can also define $z^{(p)}$, for arbitrary, not necessarily integral, $p$, by $z^{(p)}=\Gamma(z+1) / \Gamma(z-p+1)$, in terms of Euler's gamma function. We notice that the powers $z^{p}$ play a role, relative to the operator $D$, which is similar to that played by the factorials $z^{(p)}$ relative to the operator $\Delta$.

Received January 12, 1973.

AMS (MOS) subject classifications (1970). Primary 39A05, 44A45; Secondary 33A30, 33A35.

Key words and phrases. Finite difference, fractional differences, fractional derivatives, Leibniz rule, special functions, Mellin-Barnes contour integrals. 
There exists in the literature an extensive "calculus of fractional derivatives," in which the order $\alpha$ of the operator $D^{\alpha}=d^{\alpha} /(d z)^{\alpha}$ is extended to arbitrary (real or complex) numbers. To the best of the authors' knowledge, there does not exist an extensive fractional calculus for $\Delta^{\alpha}$, but only a few scattered remarks in the literature ([1], [4]). It is the purpose of this paper to inquire into the feasibility of establishing a fractional calculus of finite differences which is comparable to the already existing calculus of fractional derivatives and to investigate possible applications.

In the construction of the theory of fractional derivatives, the following three features occur:

(1) When inventing a definition for $D^{\alpha} f(z)$, for arbitrary $\alpha$, it becomes apparent that there are several alternative definitions which are not all equivalent. Thus, a particular definition must be selected, and the fractional derivative calculus developed for this choice.

(2) Rules and formulas, similar to those studied in the classical elementary calculus, can be derived, which now involve derivatives of fractional order. These include the Leibniz rule, the chain rule, Taylor's series, etc. ([6]-[13]).

(3) The fractional calculus permits us to represent the special functions of mathematical physics in a novel way. For example, the hypergeometric function of Gauss is given by

$$
{ }_{2} F_{1}\left(\begin{array}{c|c}
a, b & z \\
c & z
\end{array}\right)=\frac{\Gamma(c) z^{1-c}}{\Gamma(b)} D_{z}^{b-c} z^{b-1}(1-z)^{-a} .
$$

These fractional derivative representations, when combined with general rules and formulas described in (2), yield interesting series and integral identities involving the special functions. Thus, the fractional derivative provides another tool for investigating the properties of the special functions.

These same three features, mentioned above, also occur in constructing our theory of fractional differences. We list these three features below, and summarize the principal results of this paper.

(1) The definition we select for $\Delta^{\alpha} f(z)$ can be given in two equivalent forms, one a series, and the other an integral:

$$
\begin{aligned}
\Delta^{\alpha} f(z) & =\sum_{k=0}^{\infty}(-1)^{k}\left(\begin{array}{l}
\alpha \\
k
\end{array}\right) f(z+\alpha-k) \\
& =\frac{\Gamma(\alpha+1)}{2 \pi i} \int_{C} \frac{f(t) \Gamma(t-z-\alpha)}{\Gamma(t-z+1)} d t .
\end{aligned}
$$

The contour $C$ is shown in Fig. 2.1. This definition is discussed in Section 2.

(2) In Section 4, we derive a Leibniz rule for the fractional difference of the product of two functions

$$
\Delta^{\alpha} f(z) g(z)=\sum_{k=0}^{\infty}\left(\begin{array}{l}
\alpha \\
k
\end{array}\right) \Delta^{\alpha-k} f(z) \Delta^{k} g(z+\alpha-k) .
$$

Notice that this formula, valid for arbitrary $\alpha$, closely resembles the corresponding known formula (1.7), which is valid only for $\alpha=n=0,1,2, \cdots$. We show that, for suitably restricted functions $f(z)$ and $g(z)$, this series converges for all $z$ in a certain left half-plane. 
In proving this Leibniz rule, we make use of the following series of the GregoryNewton type:

$$
g(z)=\sum_{n=0}^{\infty} \frac{\left.\Delta^{n} g(z)\right|_{z=z_{0}-n}\left(z-z_{0}\right)_{n}}{n !}
$$

Since the authors were unable to locate this Gregory-Newton series in the literature, its derivation is given in Section 3.

(3) The fractional difference operator provides another tool for representing the special functions of mathematical physics. For example,

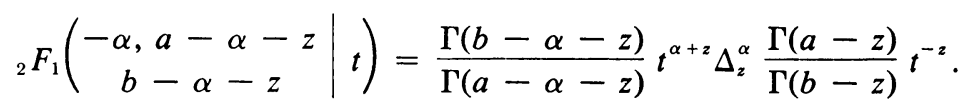

Further representations can be obtained from Table 2.1.

These fractional difference representations for the special functions, when combined with the Leibniz rule, yield series expansions relating these special functions. These are discussed in Section 5. One of the series we obtain (Example 3) is rather involved, and appeared in the literature for the first time only recently.

2. Definition of Fractional Differences. In this section, we give motivation for the concept of fractional differences, state our definition of $\Delta^{\alpha} f(z)$ precisely, and give examples of $\Delta^{\alpha} f(z)$ for specific functions $f(z)$.

We begin with motivation. Consider the following list:

$$
\begin{aligned}
\Delta f(z) & =f(z+1)-f(z) \\
\Delta^{2} f(z) & =(f(z+2)-f(z+1))-(f(z+1)-f(z)), \\
& =f(z+2)-2 f(z+1)+f(z), \\
\Delta^{3} f(z) & =f(z+3)-3 f(z+2)+3 f(z+1)-f(z), \\
\vdots & \\
\Delta^{N} f(z) & =\sum_{k=0}^{N}(-1)^{k}\left(\begin{array}{l}
N \\
k
\end{array}\right) f(z+N-k),
\end{aligned}
$$

where $N$ is a positive integer or zero. Equation (2.1) suggests that $\Delta^{\alpha} f(z)$ could be defined by the series

$$
\Delta^{\alpha} f(z)=\sum_{k=0}^{\infty}(-1)^{k}\left(\begin{array}{l}
\alpha \\
k
\end{array}\right) f(z+\alpha-k)
$$

for arbitrary $\alpha$ (rational, irrational, or complex), with the binomial coefficient defined in terms of the gamma function, provided that the series converges. Of course, (2.1) is a special case of (2.2).

Before examining (2.2) further, we give a second motivation which leads to a contour integral representation. Cauchy's integral formula, where $N$ is a positive integer or zero,

$$
D^{N} f(z)=\frac{N !}{2 \pi i} \oint \frac{f(t) d t}{(t-z)^{N+1}}
$$


suggests that, upon recalling the special meaning of $1 /(t-z)^{(N+1)}$,

$$
\Delta^{N} f(z)=\frac{N !}{2 \pi i} \oint_{C} \frac{f(t) d t}{(t-z)^{(N+1)}},
$$

since $z^{(p)}=z(z-1) \cdots(z-p+1)$ behaves, in the difference calculus, like $z^{p}$ in the differential calculus. Therefore,

$$
\begin{aligned}
\Delta^{N} f(z) & =\frac{N !}{2 \pi i} \oint_{c} \frac{f(t) d t}{(t-z)(t-z-1)(t-z-2) \cdots(t-z-N)} \\
& =\frac{N !}{2 \pi i} \oint_{C} \frac{f(t) \Gamma(t-z-N) d t}{\Gamma(t-z+1)}
\end{aligned}
$$

where $C$ encloses the simple poles $t=z+N, z+N-1, z+N-2, \cdots, z$ in the $t$-plane, and $f(t)$ is analytic inside and on $C$. Using the residue theorem, we can readily see that (2.1) and (2.3) are identical. Further, (2.3) suggests that we define for arbitrary, not necessarily integral, $\alpha$ :

$$
\Delta^{\alpha} f(z)=\frac{\Gamma(\alpha+1)}{2 \pi i} \int_{C} \frac{f(t) \Gamma(t-z-\alpha) d t}{\Gamma(t-z+1)}
$$

where $C$ is the contour shown in Fig. 2.1, enclosing the infinitely many simple poles of the integrand at $t=z+\alpha, z+\alpha-1, z+\alpha-2, \cdots$.

Before showing that (2.2) and (2.4) are identical, we investigate the possible growth of $f(z)$, for $|z|$ large an $C$, which will permit the integral in (2.4) to converge. Since

$$
\Gamma(z+a) / \Gamma(z+b) \sim z^{a-b} \text { as }|z| \rightarrow \infty,|\arg (z)|<\pi-\epsilon
$$

[5, Vol. 1, p. 33], a bound on the integrand of (2.4) for $t$ on $C$ is (using the real periodicity of the sine function, and the fact that $-t$ has a positive real part as $t \rightarrow \infty$ )

$$
\begin{aligned}
\left|\frac{f(t) \Gamma(t-z-\alpha)}{\Gamma(t-z+1)}\right| & =\left|\frac{f(t) \Gamma(z-t) \sin \pi(z-t)}{\Gamma(z+\alpha-t+1) \sin \pi(t-z-\alpha)}\right| \\
& \leqq K(z, \alpha)|f(t)|\left|(-t)^{-\alpha-1}\right|
\end{aligned}
$$

Here, the constant $K(z, \alpha)$ is independent of $t$. Thus if we assume the existence of positive constants $M$ and $p$ such that

$$
|f(t)| \leqq M\left|(-t)^{\alpha-p}\right|
$$

for $t$ on and inside the contour $C$, then

$$
\left|\frac{f(t) \Gamma(t-z-\alpha)}{\Gamma(t-z+1)}\right| \leqq M K(z, \alpha)\left|(-t)^{-p-1}\right| \leqq M K(z, \alpha)|t|^{-p-1},
$$

and thus

(i) the improper integral (2.4) is defined, and

(ii) (2.4) can be evaluated by the residue theorem.

The poles of $\Gamma(t-z-\alpha)$ occur at the points $t=z+\alpha-k$, for $k=0,1,2, \cdots$, and the corresponding residues are $(-1)^{k} / k$ !. Thus, the residue theorem applied to $(2.4)$ gives 


$$
\begin{aligned}
\frac{\Gamma(\alpha+1)}{2 \pi i} \int_{C} \frac{f(t) \Gamma(t-z-\alpha) d t}{\Gamma(t-z+1)} & =\Gamma(\alpha+1) \sum_{k=0}^{\infty} \frac{f(z+\alpha-k)(-1)^{k}}{\Gamma(\alpha-k+1) k !} \\
& =\sum_{k=0}^{\infty}(-1)^{k}\left(\begin{array}{l}
\alpha \\
k
\end{array}\right) f(z+\alpha-k)
\end{aligned}
$$

and we see that (2.2) and (2.4) are identical.

We summarize our conclusions in the following precise

Definition 2.1. Let $f(t)$ be analytic in a region $R$ containing the straight line segment $L=\{t \mid t=z+\alpha-q, 0 \leqq q\}$. Let $C$ be the contour (loop) shown in Fig. 2.1, which starts at $-\infty+i \operatorname{Im}(z+\alpha)$, encloses $L$ in the positive sense, and returns to the start without leaving $R$. Suppose also that for all complex numbers $t$ "inside" and on $C$, there exist positive constants $M$ and $p$ such that

$$
|f(t)| \leqq M\left|(-t)^{\alpha-p}\right|
$$

Then, $\Delta^{\alpha} f(z)$ is defined by (2.2) and (2.4) (both of which are shown above to be equal).

Remark: Definitions involving $\Gamma(z)$ become suspect, since the gamma function is undefined for $z=0,-1,-2, \cdots$. Looking at (2.4), we see that it contains the factor $\Gamma(\alpha+1)$, which is undefined when $\alpha$ is a negative integer. However, (2.2)

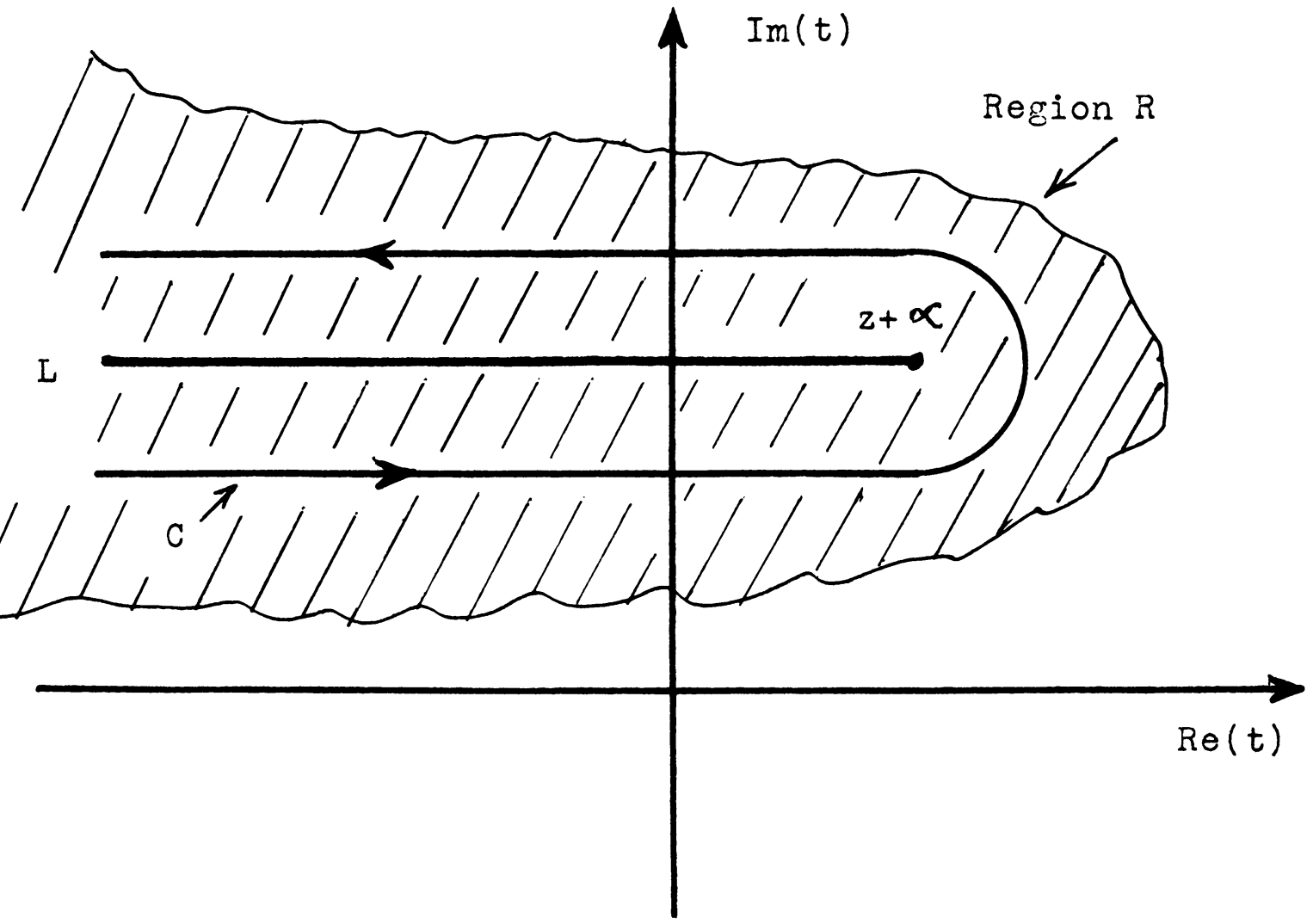

FIGURE 2.1. Contour $C$ of integration used in the definition of $\Delta a f(z)$ 
(which is equivalent to (2.4) when $\alpha$ is not a negative integer) is defined when $\alpha$ is a negative integer, because the binomial coefficient is then to be interpreted as

$$
\left(\begin{array}{l}
\alpha \\
k
\end{array}\right)=\frac{\alpha}{1} \cdot \frac{\alpha-1}{2} \cdots \frac{\alpha-k+1}{k}
$$

rather than

$$
\left(\begin{array}{l}
\alpha \\
k
\end{array}\right)=\frac{\Gamma(\alpha+1)}{\Gamma(\alpha-k+1) k !}
$$

Thus, (2.2) provides us with the analytic continuation of (2.4) to the case where $\alpha$ is a negative integer.

Another potential source of difficulty is the factor $\Gamma(t-z-\alpha)$ in the integrand of (2.4). However, this trouble is avoided by requiring that the contour $C$ not touch the line $L$, so that $t-z-\alpha$ is never zero or a negative integer.

Table 2.1 lists the fractional differences of selected functions. Item 7 of this table is of particular interest. It shows that $\Delta^{\alpha}$ is an operational device well suited to represent the contour integral which defines the $G$-function $[5$, Vol. 1, p. 144, item (4)].

Finally, we note that our above definition for fractional differences is certainly not the only reasonable one. Our definition was obtained by generalizing the formulas (2.1) and (2.3) to arbitrary nonintegral $N$. Notice, however, that if we rewrite (2.1) as

$$
\Delta^{N} f(z)=\sum_{k=0}^{N}(-1)^{N-k}\left(\begin{array}{l}
N \\
k
\end{array}\right) f(z+k)
$$

then the series definition

$$
\Delta^{\alpha} f(z)=\sum_{k=0}^{\infty}(-1)^{\alpha-k}\left(\begin{array}{l}
\alpha \\
k
\end{array}\right) f(z+k)
$$

is suggested. (2.6) is not equivalent to our definition (2.2). We will not pursue this question of alternative definitions of fractional differences in this paper, but will investigate consequences of the particular definition (2.2) studied above.

3. Gregory-Newton Type Series. Having examined the concept of fractional differences, we set it aside in this section, and investigate an expansion of the GregoryNewton type:

$$
g(z)=\sum_{n=0}^{\infty} \frac{\left.\Delta^{n} g(z)\right|_{z=z_{0}-n}\left(z-z_{0}\right)_{n}}{n !}
$$

where $(a)_{n}=a(a+1)(a+2) \cdots(a+n-1)=\Gamma(a+n) / \Gamma(a)$. We return to fractional differences in the next section, where (3.1) plays a central part in the derivation of a "Leibniz rule" for the fractional difference of a product.

We derive (3.1), as the authors have been unable to locate it in the literature.

THEOREM 3.1. Let $G(t)$ be a piecewise continuous function for $0 \leqq t$, such that

$$
|G(t)| \leqq K e^{-\gamma t}
$$

where $K$ and $\gamma$ are real constants. Let 


$$
g(z)=\int_{0}^{\infty} e^{t z} G(t) d t
$$

for $\operatorname{Re}(z)<\gamma$. Then, (3.1) is valid for $\operatorname{Re}(z)<\gamma$ and $\operatorname{Re}\left(z_{0}\right)<\gamma$.

Proof. Let $t$ be a real variable.

$$
e^{t z}=e^{t_{z 0}}\left(e^{-t}\right)^{z_{0}-z}=e^{t_{z} 0}\left(1+\left(e^{-t}-1\right)\right)^{z_{0}-z} .
$$

\begin{tabular}{|c|c|c|}
\hline & $f(z)$ & $\Delta^{\alpha} f(z)$ \\
\hline 1 & 1 & 0 \\
\hline 2 & $a^{z},|a|>1$ & $a^{z}(a-1)^{\alpha}$ \\
\hline 3 & $z^{(p)}=\frac{\Gamma(z+1)}{\Gamma(z-p+1)}$, & $\frac{\sin (\pi z) \Gamma(\alpha-p) z^{(p-\alpha)}}{\sin (\pi(z+\alpha)) \Gamma(-p)}$ \\
\hline 4 & $\frac{\Gamma(A-z)}{\Gamma(B-z)}, \quad \operatorname{Re}(B-A+\alpha)>0$ & $\frac{\Gamma(B-A+\alpha) \Gamma(A-\alpha-z)}{\Gamma(B-A) \Gamma(B-z)}$ \\
\hline 5 & $\frac{\Gamma(A-z) t^{-z}}{\Gamma(B-z)}, \quad|t|<1$ & $\frac{\Gamma(A-\alpha-z) t^{-z-\alpha}}{\Gamma(B-\alpha-z)}{ }_{2} F_{1}\left(\begin{array}{l}-\alpha, A-\alpha-z \\
B-\alpha-z\end{array} ;\right)$ \\
\hline 6 & $\begin{array}{l}\frac{\prod_{k=1}^{N} \Gamma\left(A_{k}-z\right) t^{-z}}{\prod_{k=1}^{M} \Gamma\left(B_{k}-z\right)}, \\
\qquad|t|<1, \text { for } N=M \\
\text { all } t, \text { for } N<M\end{array}$ & $\begin{array}{l}\prod_{k=1}^{N} \Gamma\left(A_{k}-\alpha-z\right) t^{-\alpha-z} \\
\underset{k=1}{M} \Gamma\left(B_{k}-\alpha-z\right) \\
N+1 F_{M}\left[\begin{array}{l}-\alpha, A_{1}-\alpha-z, \ldots, A_{N}-\alpha-z \\
B_{1}-\alpha-z, \ldots, B_{M}-\alpha-z\end{array}\right]\end{array}$ \\
\hline 7 & $\begin{array}{c}\frac{\prod_{j=1}^{m} \Gamma\left(b_{j}-z\right) t^{z}}{\prod_{j=2}^{p} \Gamma\left(a_{j}-z\right) \prod_{j=m+1}^{q-1} \Gamma\left(1-b_{j}+z\right)} \\
p \geq 1, \text { and either } p>q \\
\text { or } p=q \text { and }|t|>1\end{array}$ & 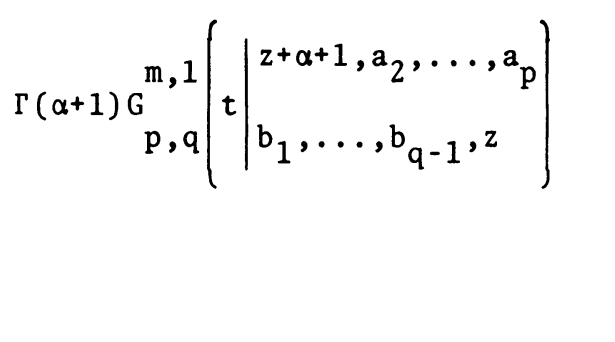 \\
\hline
\end{tabular}

TABLE 2.1 
Using the binomial theorem, that is

$$
(1+x)^{p}=\sum_{n=0}^{\infty} \frac{(-p)_{n}}{n !}(-x)^{n}
$$

where $|x|<1$, while $p$ is arbitrary, we get

$$
e^{t z}=e^{t z_{0}} \sum_{n=0}^{\infty} \frac{\left(z-z_{0}\right)_{n}\left(1-e^{-t}\right)^{n}}{n !}=\sum_{n=0}^{\infty} e^{t\left(z_{0}-n\right)}\left(e^{t}-1\right)^{n} \frac{\left(z-z_{0}\right)_{n}}{n !}
$$

for all $z$ and $z_{0}$, provided that $\left|e^{-t}-1\right|<1$, which implies that $t>\log \frac{1}{2}$, since $t$ is real. Since $\Delta_{z}{ }^{n} e^{t z}=\left(e^{t}-1\right)^{n} e^{t z}$ (see Table 2.1), we can rewrite (3.2) as

$$
e^{t z}=\sum_{n=0}^{\infty} \frac{\left.\Delta_{z}^{n} e^{t z}\right|_{z=z_{0}-n}\left(z-z_{0}\right)_{n}}{n !}
$$

for all $z$ and $z_{0}$, and for $t>\log \frac{1}{2}$.

Multiplying both sides of (3.3) by $G(t)$, and integrating from 0 to $\infty$ with respect to $t$, we get

$$
g(z)=\int_{0}^{\infty} e^{t z} G(t) d t=\int_{0}^{\infty} \sum_{n=0}^{\infty} \frac{\left.\Delta_{2}^{n} e^{t z}\right|_{z=z_{0}-n}\left(z-z_{0}\right)_{n} G(t) d t}{n !},
$$

where we note that, for $\operatorname{Re}(z)<\gamma$, the integral is finite. We shall show below that this integration can be performed termwise, and thus

$$
g(z)=\left.\sum_{n=0}^{\infty} \int_{0}^{\infty} \Delta_{z}^{n} e^{t z}\right|_{z=z_{0}-n} G(t) d t \frac{\left(z-z_{0}\right)_{n}}{n !}
$$

Since the operator $\Delta_{z}{ }^{n}$ results in a finite sum, it commutes with the operator $\int_{0}^{\infty} d t$. Thus, we get

$$
\begin{aligned}
\left.\int_{0}^{\infty} \Delta_{z}^{n} e^{t z}\right|_{z=z_{0}-n} G(t) d t & =\left.\Delta_{z}^{n}\left(\int_{0}^{\infty} e^{t z} G(t) d t\right)\right|_{z=z_{0}-n} \\
& =\left.\Delta_{z}^{n} g(z)\right|_{z=z_{0}-n} .
\end{aligned}
$$

Thus, (3.5) reduces to (3.1), and the proof will be completed-as soon as we verify the term by term integration of the series in (3.4).

Let $a_{n}(t)$ denote the general term of the series which appears under the integral sign in (3.4). It is well known that

$$
\int_{0}^{\infty} \sum_{n=0}^{\infty} a_{n}(t) d t=\sum_{n=0}^{\infty} \int_{0}^{\infty} a_{n}(t) d t
$$

provided that $\int_{0}^{\infty} \sum_{n=0}^{\infty}\left|a_{n}(t)\right| d t$ exists [15, p. 45]. Using Table 2.1, we see that

$$
\begin{aligned}
a_{n}(t) & =\left.\Delta_{z}^{n} e^{t z}\right|_{z=z_{0}-n}\left(z-z_{0}\right)_{n} G(t) / n ! \\
& =\left(e^{t}-1\right)^{n} e^{t\left(z_{0}-n\right)}\left(z-z_{0}\right)_{n} G(t) / n ! \\
& =\left(1-e^{-t}\right)^{n} e^{t z_{0}}\left(z-z_{0}\right)_{n} G(t) / n ! .
\end{aligned}
$$

Using the bound on $G(t)$, from the hypothesis, we get

$$
\left|a_{n}(t)\right| \leqq\left|K\left(1-e^{-t}\right)^{n} e^{t\left(z_{0}-\gamma\right)}\left(z-z_{0}\right)_{n} / n !\right| .
$$


But

$$
\frac{\left(z-z_{0}\right)_{n}}{n !}=\frac{\Gamma\left(z-z_{0}+n\right)}{\Gamma\left(z-z_{0}\right) \Gamma(n+1)} \sim \frac{n^{z-z_{0}-1}}{\Gamma\left(z-z_{0}\right)}
$$

as $n$ grows large [5, Vol. 1, p. 33]. Thus,

$$
\left|a_{n}(t)\right| \leqq K^{\prime} e^{t \operatorname{Re}\left(z_{0}-\gamma\right)}\left(1-e^{-t}\right)^{n} n^{\operatorname{Re}\left(z-z_{0}-1\right)}
$$

where $K^{\prime}$ is a positive constant, and we must show that $\int_{0}^{\infty} \cdots d t$ of

$$
\sum_{n=1}^{\infty}\left|a_{n}(t)\right| \leqq K^{\prime} e^{t \operatorname{Re}\left(z_{0}-\gamma\right)} \sum_{n=1}^{\infty} n^{\operatorname{Re}\left(z-z_{0}-1\right)}\left(1-e^{-t}\right)^{n}
$$

exists. The series on the right-hand side of this last expression has been studied in the literature, and we have [15, p.225]

$$
\begin{aligned}
\sum_{n=1}^{\infty} n^{\operatorname{Re}\left(z-z_{0}-1\right)} x^{n} & \left.\sim \Gamma\left(\operatorname{Re}\left(z-z_{0}\right)\right) /(1-x)^{\operatorname{Re}\left(z-z_{0}\right)}\right) & & \text { for } \operatorname{Re}\left(z-z_{0}\right)>0, \text { as } x \rightarrow 1, \\
& =-\log (1-x) & & \text { for } \operatorname{Re}\left(z-z_{0}\right)=0, \\
& <\sum_{n=1}^{\infty} n^{\operatorname{Re}\left(z-z_{0}-1\right)} & & \text { for } \operatorname{Re}\left(z-z_{0}\right)<0,
\end{aligned}
$$

where $0 \leqq x<1$. Thus, we have

$$
\begin{aligned}
\sum_{n=1}^{\infty}\left|a_{n}(t)\right| & \leqq K^{\prime} \Gamma\left(\operatorname{Re}\left(z-z_{0}\right)\right) e^{t \operatorname{Re}(z-\gamma)} & & \text { for } \operatorname{Re}\left(z-z_{0}\right)>0, \text { as } t \rightarrow+\infty, \\
& \leqq K^{\prime} t e^{t \operatorname{Re}\left(z_{0}-\gamma\right)} & & \text { for } \operatorname{Re}\left(z-z_{0}\right)=0, \\
& \leqq \sum_{n=1}^{\infty} n^{\operatorname{Re}\left(z^{2}-z_{0}-1\right)} e^{t \operatorname{Re}\left(z_{0}-\gamma\right)} & & \text { for } \operatorname{Re}\left(z-z_{0}\right)<0,
\end{aligned}
$$

for $0 \leqq t$. Since $\operatorname{Re}(z-\gamma)$ and $\operatorname{Re}\left(z_{0}-\gamma\right)$ are negative, by hypothesis, we can integrate this last expression from 0 to $\infty$ in $t$, and the theorem is proved.

An interesting example. The theorem just proved tells us that (3.1) is true, provided $\operatorname{Re}(z)$ and $\operatorname{Re}\left(z_{0}\right)<\gamma$. Are both these conditions necessary? It is of interest to examine a special case of (3.1) to gain some insight into the meaning of these restrictions. If we select $G(t)=e^{-\gamma t}$, where $\gamma$ is real, then, for $\operatorname{Re}(z)<\gamma$,

$$
g(z)=\int_{0}^{\infty} e^{z t} G(t) d t=\frac{1}{\gamma-z}=\frac{\Gamma(\gamma-z)}{\Gamma(\gamma-z+1)} .
$$

Substituting this value of $g(z)$ into (3.1), we get (after some simplification and the use of Table 2.1)

$$
\frac{1}{\gamma-z}=\frac{1}{\gamma-z_{0}} \sum_{n=0}^{\infty} \frac{\left(z-z_{0}\right)_{n}}{\left(\gamma-z_{0}+1\right)_{n}}={ }_{2} F_{1}\left[\begin{array}{c|c}
z-z_{0}, 1 \\
\gamma-z_{0}+1
\end{array} \mid 1\right] .
$$

The right-hand side converges absolutely provided $\operatorname{Re}(z)<\gamma$, and can be summed [5, p. 99] to yield the left-hand side. Thus, we see that the condition $\operatorname{Re}(z)<\gamma$ is needed for the convergence of the series (3.1) in this example. The condition $\operatorname{Re}\left(z_{0}\right)<\gamma$ seems to be important only to insure that $g(z)$ is defined by the integral above. 
We will not pursue this question of the importance of the restrictions listed in Theorem 3.1 further. Rather, we use this result as a basis to prove the Leibniz rule for fractional differences in the next section.

4. The Leibniz Rule for Fractional Differences. In this section, we derive the Leibniz rule for fractional differences

$$
\Delta^{\alpha} f(z) g(z)=\sum_{n=0}^{\infty}\left(\begin{array}{l}
\alpha \\
n
\end{array}\right) \Delta^{\alpha-n} f(z) \Delta^{n} g(z+\alpha-n) .
$$

The form of (4.1) is readily anticipated when we recall the known form of the Leibniz rule,

$$
\Delta^{N} f(z) g(z)=\sum_{n=0}^{N}\left(\begin{array}{l}
N \\
n
\end{array}\right) \Delta^{N-n} f(z) \Delta^{n} g(z+N-n),
$$

familiar from the study of finite differences, in which $N=0,1,2, \cdots$.

THEOREM 4.1. Let $G(s)$ be a piecewise continuous function for $0 \leqq s$ such that

(i) $|G(s)| \leqq M e^{-\gamma s}$, where $\gamma$ is real.

Let $g(z)$ be defined by

$$
g(z)=\int_{0}^{\infty} e^{s z} G(s) d s, \quad \text { for } \quad \operatorname{Re}(z)<\gamma .
$$

Let $z$ and $\alpha$ be fixed numbers such that

$$
\operatorname{Re}(z+\alpha) \leqq \gamma-1 .
$$

Let $R$ be a region in the t-plane containing the horizontal semi-infinite straight line segment $L=\{t \mid t=z+\alpha-q, 0 \leqq q\}$. Let $f(t)$ be analytic on $R$, and let

$$
|f(t)| \leqq K\left|(-t)^{\alpha+1-p}\right| e^{\operatorname{Re}(t)}
$$

for fixed positive constants $K$ and $p$, and all $t$ in $R$. Then the Leibniz rule for fractional differences (4.1) is valid.

Proof. Replace $z_{0}$ in Theorem 3.1 by $z+\alpha$, and replace $z$ by $t$. We have, then, for $\operatorname{Re}(z+\alpha)<\gamma$ and $\operatorname{Re}(t)<\gamma$

$$
g(t)=\sum_{n=0}^{\infty}\left(\Delta^{n} g(z+\alpha-n)\right)(t-z-\alpha)_{n} / n ! .
$$

Multiply both sides of this relation by

$$
\frac{\Gamma(\alpha+1)}{2 \pi i} \frac{f(t) \Gamma(t-z-\alpha)}{\Gamma(t-z+1)}
$$

and obtain

$$
\begin{aligned}
& \frac{\Gamma(\alpha+1)}{2 \pi i} \frac{f(t) g(t) \Gamma(t-z-\alpha)}{\Gamma(t-z+1)} \\
& \quad=\sum_{n=0}^{\infty} \frac{\Delta^{n} g(z+\alpha-n)}{n !} \frac{\Gamma(\alpha+1)}{\Gamma(\alpha-n+1)} \frac{\Gamma(\alpha-n+1)}{2 \pi i} \frac{f(t) \Gamma(t-z-\alpha+n)}{\Gamma(t-z+1)} .
\end{aligned}
$$

Let $C$ be the contour of integration shown in Figure 2.1 (and described in Definition 
2.1 of $\Delta^{\alpha} f(z)$ ). If we integrate both sides of (4.2) over $C$ (with respect to $t$ ), and assume that (i) the integrals exist, and (ii) we can integrate the right-hand side of (4.2) term by term, then we obtain the desired Leibniz rule at once.

It is clear that the integrals just mentioned exist, as follows from the discussion in Definition 2.1, together with items (i) through (iv) of the hypothesis. Let $u_{n}(t)$ denote the $n$th term on the right-hand side of (4.2). If we can show that $\int_{0}^{\infty} \sum_{n=n}^{\infty}\left|u_{n}(t)\right||d t|$ exists, then we know that term by term integration is permissible $[15$, p. 45$]$.

To this end, we first estimate

$$
\begin{aligned}
\left|\Delta^{n} g(z+\alpha-n)\right| & =\left|\Delta^{n} \int_{0}^{\infty} e^{(z+\alpha-n) s} G(s) d s\right| \\
& =\left|\int_{0}^{\infty} \Delta_{z}^{n} e^{(z+\alpha-n) s} G(s) d s\right| \\
& =\left|\int_{0}^{\infty}\left(e^{s}-1\right)^{n} e^{(z+\alpha-n) s} G(s) d s\right| \\
& \leqq M \int_{0}^{\infty}\left(1-e^{-s}\right)^{n} e^{\operatorname{Re}(z+\alpha-\gamma) s} d s .
\end{aligned}
$$

Using item (iii) of the hypothesis, we get

$$
\left|\Delta^{n} g(z+\alpha-n)\right| \leqq M \int_{0}^{\infty}\left(1-e^{-s}\right)^{n} e^{-s} d s=M /(n+1) .
$$

Now, it is clear that

$$
\begin{aligned}
\sum_{n=0}^{\infty} \mid & u_{n}(t) \mid \\
& \leqq \sum_{n=0}^{\infty}\left|\frac{\Gamma(\alpha+1)}{2 \pi} \frac{f(t) \Gamma(t-z-1+\alpha)}{\Gamma(t-z+1)} \frac{(t-z-1-\alpha)_{n+1}}{n !} \frac{M}{(n+1)}\right| \\
& \leqq \frac{|\Gamma(\alpha+1)| M}{2 \pi}\left|\frac{f(t) \Gamma(t-z-1-\alpha)}{\Gamma(t-z+1)}\right| \sum_{n=0}^{\infty}\left|\frac{(t-z-1-\alpha)_{n+1}}{(n+1) !}\right| .
\end{aligned}
$$

It is well known that this last series converges if $\operatorname{Re}(t)<1+\operatorname{Re}(z+\alpha)[5, \mathrm{pp} .43$, 44]. This inequality shows that the series in (4.3) converge for each $t$ on the contour $C$. Thus, we can integrate over $C$, provided only that this last expression tends to zero sufficiently fast on $C$ as $\operatorname{Re}(t) \rightarrow-\infty$. It remains, then, to estimate

$$
S=\sum_{n=0}^{\infty}\left|(t-z-1-\alpha)_{n+1} /(n+1) !\right|
$$

when $\operatorname{Re}(-t)$ is large.

To this end, keep the contour $C$ between the two lines

$$
\operatorname{Im}(t)=\operatorname{Im}(z+\alpha) \pm 1
$$

Let $N$ be a natural number

$$
3 \leqq N \text {, }
$$

and consider only complex numbers $t$ on the contour $C$ such that 


$$
1-N \leqq \operatorname{Re}(t-z-\alpha)<2-N .
$$

The relations (4.4a) and (4.4c) confine $t$ to a rectangle in the complex plane, having base 1 and altitude 2 (see Fig. 4.1). By increasing $N$, (4.4c) will permit us to consider $t$ further and further to the left on the contour $C$. Now, the sequence of numbers (see Fig. 4.2) $\left\{a_{k}\right\}=\{|t-z-\alpha-1+k|\}_{k=0}^{\infty}$ is such that $a_{0}>a_{1}>a_{2}>\cdots>$ $a_{N-1}$, and $a_{N}<a_{N+1}<a_{N+2}<\cdots$, because (4.4c) implies that

$$
0 \leqq \operatorname{Re}(t-z-\alpha-1+N)<1
$$

Thus

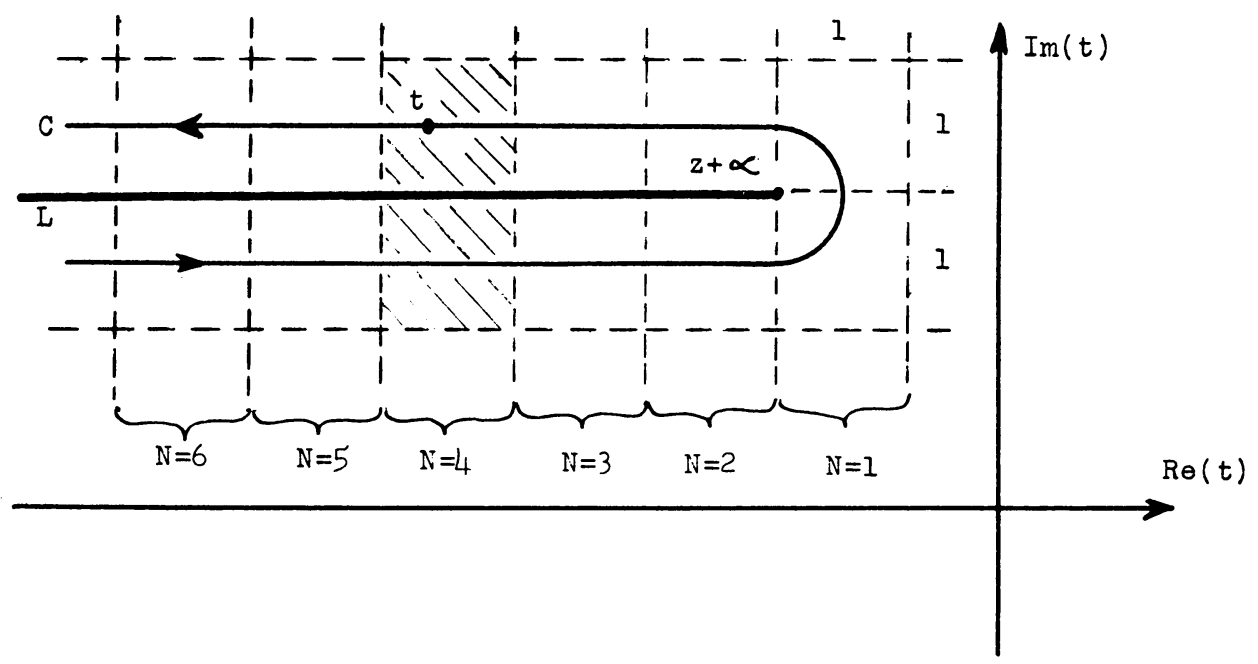

FIGURE 4.1. Geometry of relations (4.4a), (4.4b), and (4.4c), with t shown in the block $N=4$.
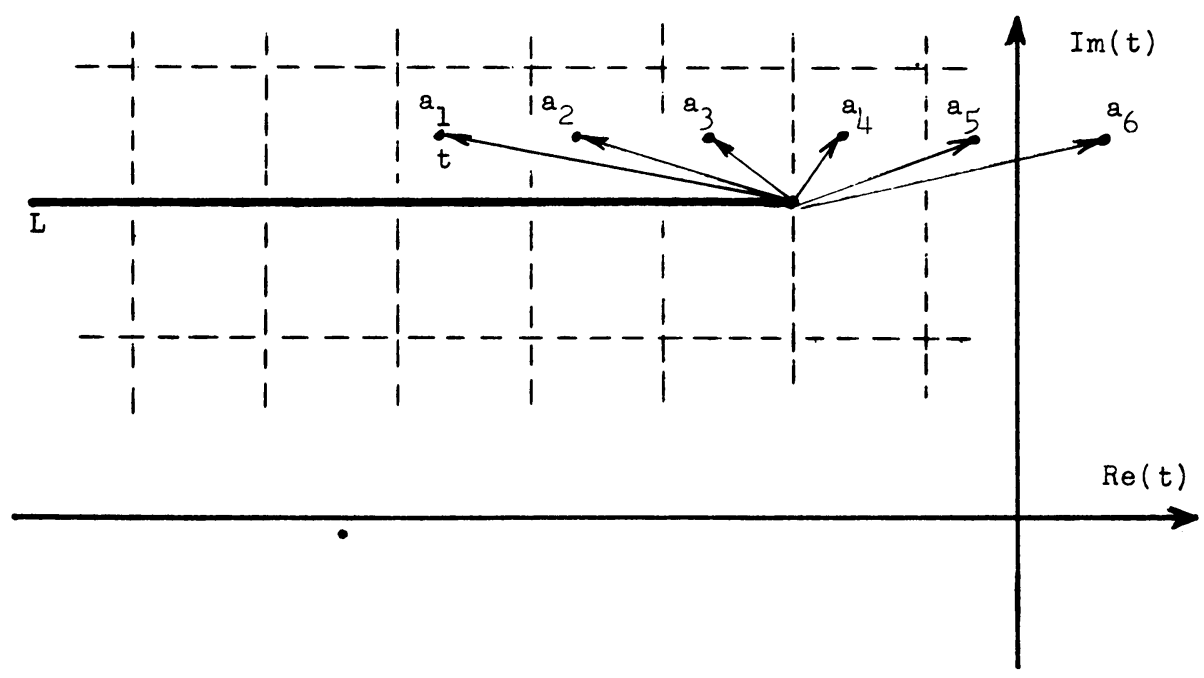

FIGURE 4.2. The geometry of the sequence $a_{1}, a_{2}, a_{3}, \cdots$ 


$$
\begin{aligned}
S= & \sum_{n=0}^{\infty}\left|\frac{(t-z-\alpha-1)_{n}}{n !}\right|=\sum_{n=0}^{N}(\cdots)+\sum_{n=N+1}^{\infty}(\cdots) \\
= & \sum_{n=0}^{N} \frac{\left|(t-z-\alpha-1)_{n}\right|}{n !}+\frac{\left|(t-z-\alpha-1)_{N}\right|}{N !} \sum_{r=1}^{\infty} \frac{\left|(t-z-\alpha-1+N)_{r}\right|}{(N+1)_{r}} \\
\leqq & \sum_{n=0}^{N} \frac{(1-\operatorname{Re}(t-z-\alpha-1))^{n}}{n !}+\frac{\left|(t-z-\alpha-1)_{N}\right|}{N !} \\
& \cdot \sum_{r=1}^{\infty} \frac{(1+\operatorname{Re}(t-z-\alpha-1+N))_{r}}{(N+1)_{r}},
\end{aligned}
$$

where we have used

$$
\begin{aligned}
1-\operatorname{Re}(t-z-\alpha-1) & \geqq|t-z-\alpha-1| \\
& \geqq|t-z-\alpha| \geqq \cdots \geqq|t-z-\alpha-2+N|
\end{aligned}
$$

in the first series, and have used

$$
1+\operatorname{Re}(t-z-\alpha-1+N+k)>|(t-z-\alpha-1+N+k)|
$$

in the second series, $k=0,1,2, \cdots$. The first series in this last expression is a partial sum of the exponential series, while the second is of hypergeometric type:

$$
1+\sum_{n=1}^{\infty} \frac{(a)_{n}(b)_{n}}{(c)_{n} n !}=\frac{\Gamma(c) \Gamma(c-a-b)}{\Gamma(c-a) \Gamma(c-b)} \text { where } b=1
$$

Therefore,

$$
\begin{aligned}
& S<\exp (2-\operatorname{Re}(t-z-\alpha))+\frac{\left|(t-z-\alpha-1)_{N}\right|}{N !} \frac{\Gamma(N+1) \Gamma(-\operatorname{Re}(t-z-\alpha))}{\Gamma(N) \Gamma(1-\operatorname{Re}(t-z-\alpha))}, \\
& S<\exp (2-\operatorname{Re}(t-z-\alpha))+\frac{(2-\operatorname{Re}(t-z-\alpha))^{N}}{N !} \frac{N}{(-\operatorname{Re}(t-z-\alpha))}, \\
& S<\exp (2-\operatorname{Re}(t-z-\alpha))+\frac{(2-\operatorname{Re}(t-z-\alpha))}{(-\operatorname{Re}(t-z-\alpha))} \frac{(2-\operatorname{Re}(t-z-\alpha))^{N-1}}{(N-1) !} .
\end{aligned}
$$

Looking at the second term in this last expression, (4.4b) and (4.4c) imply that

$$
0<\frac{2-\operatorname{Re}(t-z-\alpha)}{-\operatorname{Re}(t-z-\alpha)} \leqq \frac{N+1}{N-2} \leqq 4,
$$

and since $x^{N-1} /(N-1) !<e^{x}$ for $x>0$, we get

$$
S<\exp (2-\operatorname{Re}(t-z-\alpha))+4 \exp (2-\operatorname{Re}(t-z-\alpha)) .
$$

Therefore,

$$
S<5 \exp (2-\operatorname{Re}(t-z-\alpha))
$$

But this last relation is independent of $N$, and (4.4b) shows that this argument is true regardless of how large $\operatorname{Re}(-t)$ becomes.

Returning to (4.3), we substitute this last estimate, and obtain 


$$
\begin{aligned}
\sum_{n=0}^{\infty}\left|u_{n}(t)\right| \leqq & \frac{5 M|\Gamma(\alpha+1)|}{2 \pi}\left|\frac{f(t) \Gamma(t-z-\alpha-1)}{\Gamma(t-z+1)}\right| \\
& \cdot \exp (2-\operatorname{Re}(t-z-\alpha)) .
\end{aligned}
$$

In our discussion of Definition 2.1, we saw that

$$
|\Gamma(t-z-\alpha-1) / \Gamma(t-z+1)| \leqq K^{\prime}\left|(-t)^{-\alpha-2}\right|
$$

for $t$ on $C$ ( $K^{\prime}$ independent of $\left.t\right)$. Substituting this last relation and item (iv) of the hypothesis into (4.5), we get

$$
\sum_{n=0}^{\infty}\left|u_{n}(t)\right| \leqq M^{\prime \prime}\left|(-t)^{-1-p}\right|,
$$

where the constant $M^{\prime \prime}$ does not depend upon $t$. Thus

$$
\int_{0}^{\infty} \sum_{n=0}^{\infty}\left|u_{n}(t)\right||d t|
$$

exists, and the theorem is proved.

In the next section, we will derive several interesting results from our Leibniz rule (4.1), by simply selecting specific functions for $f(z)$ and $g(z)$. However, we note at this time that items (i) and (ii) of the hypothesis of Theorem 4.1 may be difficult to check. This is because, after first selecting $g(z)$, we must find $G(s)$ such that

$$
g(z)=\int_{0}^{\infty} e^{s z} G(s) d s,
$$

and finally we must investigate $G(s)$ to determine the critical constant $\gamma$ such that $|G(s)| \leqq M e^{-\gamma}$. Fortunately, the existence of $G(s)$ and its growth have been studied in the literature [14, p. 30]. Using this reference, we see at once that items (i) and (ii) in the hypothesis of Theorem 4.1 can be replaced by items (i) and (ii) in the corollary below. Also, item (iv) can be replaced by

$$
|f(t)| \leqq K e^{c \operatorname{Re}(t)}, \quad c>1,
$$

which is stronger than (iv), but better suited for applications.

We summarize our conclusions in

COROLlaRY 4.1. Let

(i) $g(z)$ be analytic for $\operatorname{Re}(z)<\gamma$; and

(ii) $|g(z)| \leqq M\left|z^{-k}\right|$ for $\operatorname{Re}(z)<\gamma$, where $M>0$ and $k>1$; and

(iii) $f(t)$ be analytic on $R$ (defined in Theorem 4.1); and

(iv) $|f(t)| \leqq K e^{\mathrm{c} R e(t)}$ for some fixed $K>0$ and $c>1$, and all $t$ in $R$.

Then the Leibniz rule for fractional differences (4.1) is valid for

(v) $\operatorname{Re}(z+\alpha)<\gamma-1$.

5. Applications to Infinite Series. In this section, specific functions $f(z)$ and $g(z)$ are selected for use in our new Leibniz rule (4.1). Table 2.1 is used to compute the differences $\Delta^{\alpha} f g, \Delta^{\alpha-n} f$, and $\Delta^{n} g$ encountered. Several interesting results are found to be special cases of our Leibniz rule for fractional differences.

Example 1. Let $f(z)=w^{-z}$ and $g(z)=\Gamma(a-z) / \Gamma(b-z), a$ and $b$ real, and $w$ positive. Substituting directly into (4.1) and using Table 2.1, we get, after minor simplification, 


$$
{ }_{2} F_{1}\left[\begin{array}{c|c}
-\alpha, a-z-\alpha & w \\
b-z-\alpha &
\end{array}\right]=(1-w)^{\alpha} \sum_{n=0}^{\infty} \frac{(-\alpha)_{n}(b-a)_{n}}{(b-z-\alpha)_{n} n !}\left(\frac{w}{w-1}\right)^{n} .
$$

Expressing the right-hand side as a hypergeometric function ${ }_{2} F_{1}$, we get the known result $[5$, p. $67,(2)]$

$$
{ }_{2} F_{1}\left[\begin{array}{c|c}
-\alpha, a-z-\alpha & w \\
b-z-\alpha &
\end{array}\right]=(1-w)_{2}^{\alpha} F_{1}\left[\begin{array}{c|c}
-\alpha, b-a & w \\
b-z-\alpha & w-1
\end{array}\right] .
$$

Next, let us examine the restrictions imposed by Corollary 4.1. Item (i) reveals that $\gamma=a$. From the relation

$$
g(z)=\Gamma(a-z) / \Gamma(b-z) \sim(-z)^{a-b}
$$

so often used in this paper, we see that (ii) is satisfied if we take $a-b<-1$. Items (iii) and (iv) can be satisfied by taking $w<e^{-1}$ and item (v) by $\operatorname{Re}(z)<-\operatorname{Re}(\alpha)+$ $a-1$. It is clear from (5.1) that these restrictions are sufficient for the convergence of the series, but it is also clear that they are too strong since the series converges for $|w /(w-1)|<1$, which implies that $\operatorname{Re}(w)<\frac{1}{2}$.

Example 2. Looking at our Leibniz rule (4.1), it is quite clear that we can interchange $f(z)$ and $g(z)$ on the left-hand side, but this is not clear on the right-hand side. In fact, very different restrictions are imposed on $f(z)$ and $g(z)$ in Corollary 4.1. Let us experiment, and interchange $f$ and $g$ in the preceding example. Thus, we take $f(z)=\Gamma(a-z) / \Gamma(b-z)$ and $g(z)=w^{-z}$. Substituting directly into (4.1), using Table 2.1, and simplifying, we get

$$
\begin{aligned}
& \frac{\Gamma(a-\alpha-z) w^{-z-\alpha}}{\Gamma(b-\alpha-z)}{ }_{2} F_{1}\left[\begin{array}{c|c}
-\alpha, a-\alpha-z & w \\
b-\alpha-z & \mid
\end{array}\right] \\
& \quad=\sum_{n=0}^{\infty} \frac{(-\alpha)_{n}}{n !} \frac{\pi \Gamma(a-\alpha-z+n)\left(w^{-1}-1\right)^{n} w^{-z-\alpha+n}}{\Gamma(b-a) \Gamma(b-z) \sin \pi(b-a+\alpha) \Gamma(1+a-b-\alpha+n)} .
\end{aligned}
$$

That is,

$$
\begin{aligned}
{ }_{2} F_{1}\left[\begin{array}{r|r}
-\alpha, a-\alpha-z & w \\
b-\alpha-z &
\end{array}\right] \\
\quad=\frac{\Gamma(b-\alpha-z) \Gamma(b-a+\alpha)}{\Gamma(b-a) \Gamma(b-z)} \sum_{n=0}^{\infty} \frac{(-\alpha)_{n}(a-\alpha-z)_{n}}{(a-b-\alpha+1)_{n} n !}(1-w)^{n} \\
\quad=\frac{\Gamma(b-a+\alpha) \Gamma(b-\alpha-z)}{\Gamma(b-a) \Gamma(b-z)}{ }_{2} F_{1}\left[\begin{array}{l}
-\alpha, a-\alpha-z \\
a-b-\alpha+1
\end{array} \mid 1-w\right]
\end{aligned}
$$

which is "one half" of the correct identity [5, Vol. 1, p. 70, (6)], unless $\alpha$ is a positive integer or zero. Thus, we see that violating the hypothesis of Corollary 4.1 may lead to wrong results.

Example 3. If we take

$$
f(z)=\frac{\prod_{k=1}^{p-1} \Gamma\left(a_{k}^{\prime}-z\right)}{\prod_{k=1}^{a} \Gamma\left(b_{k}^{\prime}-z\right)} x^{-z} \frac{\prod_{k=1}^{\prime} \Gamma\left(\alpha_{k}^{\prime}-z\right)}{\prod_{k=1}^{u} \Gamma\left(\beta_{k}^{\prime}-z\right)}
$$

and 


$$
g(z)=\frac{\prod_{k=1}^{r} \Gamma\left(c_{k}^{\prime}-z\right)}{\prod_{k=1}^{s} \Gamma\left(d_{k}^{\prime}-z\right)} y^{-z} \frac{\prod_{k=1}^{u} \Gamma\left(\beta_{k}^{\prime}-z\right)}{\prod_{k=1}^{t} \Gamma\left(\alpha_{k}^{\prime}-z\right)}
$$

in our Leibniz rule (4.1), we obtain

$$
\begin{aligned}
{ }_{p+r} F_{q+s}\left[\begin{array}{c}
-\alpha, a_{1}, \cdots, a_{p-1}, c_{1}, \cdots, c_{r} \\
b_{1}, \cdots, b_{q}, d_{1}, \cdots, d_{s}
\end{array}\right] \\
=\sum_{n=0}^{\infty} \frac{(-1)^{n}(-\alpha)_{n}}{n !} \frac{\prod_{k=1}^{p-1}\left(a_{k}\right)_{n} \prod_{k=1}^{t}\left(\alpha_{k}\right)_{n} x^{n}}{\prod_{k=1}^{a}\left(b_{k}\right)_{n} \prod_{k=1}^{u}\left(\beta_{k}\right)_{n}} \\
\cdot_{{ }^{+}+\iota} F_{q+u}\left[\begin{array}{c}
-\alpha+n, a_{1}+n, \cdots, a_{p-1}+n, \alpha_{1}+n, \cdots, \alpha_{t}+n \mid x \\
b_{1}+n, \cdots, b_{q}+n, \beta_{1}+n, \cdots, \beta_{u}+n
\end{array}\right] \\
\cdot_{1+r+u} F_{s+\iota}\left[\begin{array}{c}
-n, c_{1}, \cdots, c_{r}, \beta_{1}, \cdots, \beta_{u} \mid y \\
d_{1}, \cdots, d_{s}, \alpha_{1}, \cdots, \alpha_{t}
\end{array}\right]
\end{aligned}
$$

where we have used the condensed notation $a_{k}=a_{k}{ }^{\prime}-\alpha-z, b_{k}=b_{k}{ }^{\prime}-$ $\alpha-z, \cdots$, etc.

To obtain the restrictions imposed on (5.2) by Corollary 4.1, we use the wellknown asymptotic approximation [5, Vol. 1, p. 32, (2)]

$$
\Gamma(z+a) \sim(2 \pi)^{1 / 2} e^{-z} z^{(z+a)-1 / 2}
$$

as $|z| \rightarrow \infty$ and $|\arg (z)| \leqq \pi-\epsilon, \epsilon>0$. Substituting (5.3) into the definitions of $f(z)$ and $g(z)$, we obtain

$$
\begin{aligned}
f(z) \sim & (2 \pi)^{(p+t-q-u-1) / 2} e^{(p+t-q-u-1) z} x^{-z} \\
& \cdot(-z)^{(-z-1 / 2)(p+t-q-u-1)+\sum_{a k^{\prime}}+\sum_{\alpha_{k^{\prime}}} \sum_{b_{k^{\prime}}}-\sum_{\beta_{k^{\prime}}}}
\end{aligned}
$$

and

$$
\begin{aligned}
g(z) \sim & (2 \pi)^{(r+u-s-t) / 2} e^{(r+u-s-t) z} y^{-z} \\
& \cdot(-z)^{(-z-1 / 2)(r+u-s-t)+\sum_{c k^{\prime}}+\sum_{\beta_{k^{\prime}}}-\sum_{d_{k^{\prime}}}-\sum_{\alpha_{k^{\prime}}}}
\end{aligned}
$$

as $|z| \rightarrow \infty$ and $|\arg (-z)|<\pi-\epsilon, \epsilon>0$. Thus, we see that the terms of the form $(-z)^{P(-z)}$ dominate these asymptotic expansions. We see, from Corollary 4.1 , that we require

$$
p+t-q-u-1<0 \text { and } r+u-s-t<0 .
$$

We also see that $x$ and $y$ are arbitrary, and that we should take

$$
\operatorname{Re}(z+\alpha)<\gamma+1
$$

where

$$
\gamma=\operatorname{Min}\left\{\operatorname{Re}\left(a_{k}^{\prime}\right), \operatorname{Re}\left(\alpha_{k}^{\prime}\right), \operatorname{Re}\left(c_{k}^{\prime}\right), \operatorname{Re}\left(\beta_{k}^{\prime}\right) ; k=1,2, \cdots\right\} .
$$

This series (5.2) can be found in [5, Vol. 2, p. 11, (27)]. Our restrictions (5.4) do not seem to be given in the list in this reference.

Example 4. If we select 


$$
f(z)=\frac{\prod_{k=1}^{m} \Gamma\left(b_{k}-z\right) x^{z}}{\prod_{k=2}^{p} \Gamma\left(a_{k}-z\right) \prod_{k=m+1}^{a-1} \Gamma\left(1-b_{k}+z\right)} \frac{\prod_{k=1}^{R} \Gamma\left(\beta_{k}-z\right)}{\prod_{k=1}^{s} \Gamma\left(\alpha_{k}-z\right)}
$$

and

$$
g(z)=\frac{\prod_{k=1}^{M} \Gamma\left(B_{k}-z\right) y^{z}}{\prod_{k=2}^{P} \Gamma\left(A_{k}-z\right) \prod_{k=M+1}^{Q-1} \Gamma\left(1-B_{k}+z\right)} \frac{\prod_{k=1}^{S} \Gamma\left(\alpha_{k}-z\right)}{\prod_{k=1}^{R} \Gamma\left(\beta_{k}-z\right)}
$$

for use in our Leibniz rule (4.1), we obtain

$$
\begin{aligned}
& G_{p+P-1, Q+Q-1}^{m+M, 1}\left(x y \mid \begin{array}{c}
z+\alpha+1, a_{2}, \cdots, a_{p}, A_{2}, \cdots, A_{p} \\
b_{1}, \cdots, b_{q-1}, B_{1}, \cdots, B_{Q-1}, z
\end{array}\right) \\
& =\sum_{n=0}^{\infty} G_{p+S, q+R}^{m+R, 1}\left(x \mid \begin{array}{c}
z+\alpha-n+1, a_{2}, \cdots, a_{p}, \alpha_{1}, \cdots, \alpha_{S} \\
b_{1}, \cdots, b_{m}, \beta_{1}, \cdots, \beta_{R}, b_{m+1}, \cdots, b_{a-1}, z
\end{array}\right) \\
& \cdot G_{P+R, Q+S}^{M+S, 1}\left(y \mid \begin{array}{c}
z+\alpha+1, A_{2}, \cdots, A_{p}, \beta_{1}, \cdots, \beta_{R} \\
B_{1}, \cdots, B_{M}, \alpha_{1}, \cdots, \alpha_{S}, B_{M+1}, \cdots, B_{Q-1}, z+\alpha-n
\end{array}\right) .
\end{aligned}
$$

(5.5) is a "multiplication theorem" for the $G$-functions.

Again, we use (5.3) to determine the nature of $f(z)$ and $g(z)$ as $z$ grows large. We find that the asymptotic expansions for $f(z)$ and $g(z)$ are dominated by the terms $(-z)^{(-z)(Q+R-p-S)}$ and $(-z)^{(-z)(Q+S-P-R)}$, respectively, where $|z| \rightarrow \infty$ and $|\arg (-z)|$ $\leqq \pi-\epsilon, \epsilon>0$. Thus we see from items (ii) and (iv) of Corollary 4.1 , that we have the restrictions

$$
q+R-p-S<0 \text { and } Q+S-P-R<0 .
$$

From items (i), (iii) and (v) of that corollary, we see that we must take $\operatorname{Re}(z+\alpha)<$ $\gamma-1$, where $\gamma=\operatorname{Min}\left\{b_{k}, B_{k}, \alpha_{k}, \beta_{k}\right\}$.

6. Concluding Remark. This paper has provided only a brief exploratory journey into the yet undiscovered area of fractional differences. In fact, we seem to raise more questions than we provide answers. In particular, we note that only one of several possible definitions for $\Delta^{\alpha} f(z)$ has been used throughout this paper. Alternative definitions remain to be studied and their interrelationship clarified. It would also be of interest to study a fractional calculus involving an arbitrary span " $h$ ", that is to say, where $\Delta f(z)=f(z+h)-f(z)$, and to examine the natural limit $\lim _{h \rightarrow 0} \Delta^{\alpha} f(z) / h^{\alpha}$ and its relation to $D^{\alpha} f(z)$. Examination of natural rules, such as the "law of exponents" $\Delta^{\alpha} \Delta^{\beta}=\Delta^{\alpha+\beta}$, the chain rule, etc., remain to be performed.

Department of Mathematics

Rensselaer Polytechnic Institute

Troy, New York 12181

Department of Mathematics

Glassboro State College

Glassboro, New Jersey 08028

1. G. T. CARgo \& O. Shisha, "Zeros of polynomials and fractional order differences of their coefficients," J. Math. Anal. Appl., v. 7, 1963, pp. 176-182. MR 28 \#2195. 
2. E. T. Copson, An Introduction to the Theory of Functions of a Complex Variable, Oxford Univ. Press, Oxford, 1935.

3. H. T. Davis, The Summation of Series, Principia Press of Trinity Univ., San Antonio, Texas, 1962. MR 25 \#5305.

4. B. Kuttner, "On differences of fractional order," Proc. London Math. Soc., (3), v. 7, 1957, pp. 453-466. MR 20 \#1131.

5. Y. L. Luke, The Special Functions and Their Approximations. Vols. I, II, Math. in Sci. and Engineering, vol. 53, Academic Press, New York, 1969. MR 39 \#3039; MR 40 \#2909.

6. T. J. OsLER, "Leibniz rule for fractional derivatives generalized and an application to infinite series," SIAM J. Appl. Math., v. 18, 1970, pp. 658-674.

7. T. J. OSLER, "The fractional derivative of a composite function," SIAM J. Math Anal., v. 1, 1970, pp. 288-293. MR 41 \#5563.

8. T. J. OsLER, "Taylor's series generalized for fractional derivatives and applications," SIAM J. Math. Anal., v. 2, 1971, pp. 37-48. MR 45 \#3682.

9. T. J. OsLer, "Fractional derivatives and Leibniz rule," Amer. Math. Monthly, v. 78, 1971 , pp. $645-649$.

10. T. J. OSLER, "A further extension of the Leibniz rule to fractional derivatives and its relation to Parseval's formula," SIAM J. Math. Anal., v. 3, 1972, pp. 1-16.

11. T. J. OsLER, "An integral analogue of Taylor's series and its use in computing Fourier transforms," Math. Comp., v. 26, 1972, pp. 449-460.

12. T. J. OSLER, "A correction to Leibniz rule for fractional derivatives," SIAM J. Math. Anal., v. 4, 1973, pp. 456-459.

13. T. J. OSLER, "The integral analog of the Leibniz rule," Math. Comp., v. 26, 1972, pp. 903-915.

14. I. N. Sneddon, Fourier Transforms, McGraw-Hill, New York, 1951. MR 13, 29. 1939.

15. E. C. Titchmarsh, The Theory of Functions, 2nd ed., Oxford Univ. Press, Oxford, 